\title{
A influência dos alimentos afrodisíacos no desejo sexual: revisão integrativa
}

\author{
The influence of aphrodisiac foods in sexual desire: integrative review \\ La influencia de losalimentos afrodisiacos en el deseo sexual: revisión integrativa
}

Recebido: 04/11/2021 | Revisado: 19/11/2021 | Aceito: 04/12/2021 | Publicado: 14/12/2021

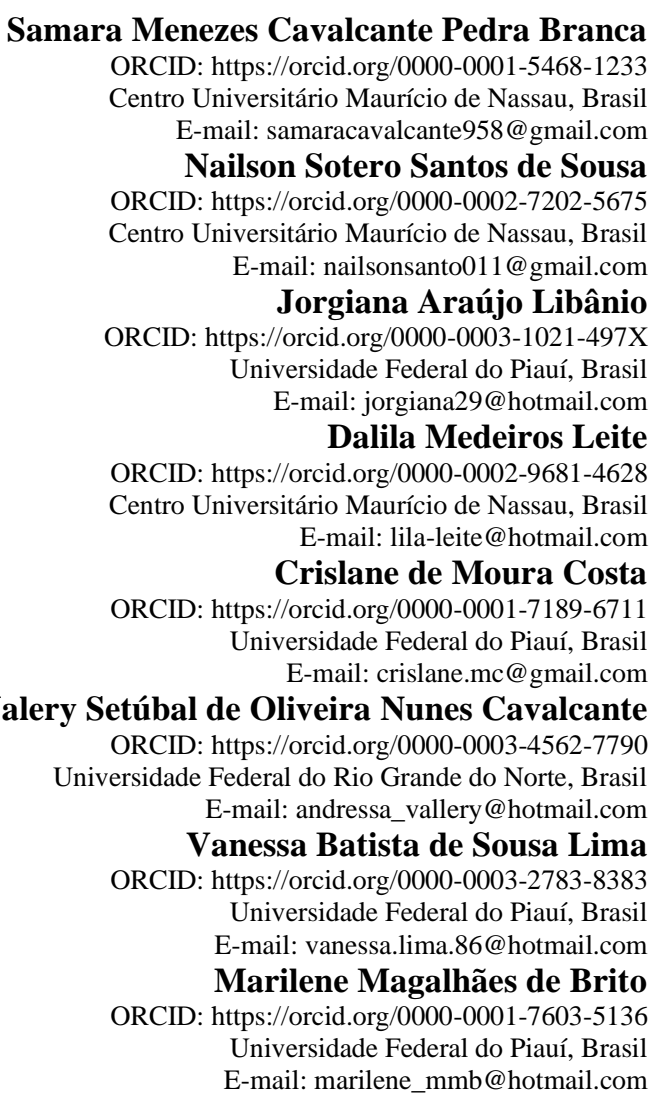

\begin{abstract}
Resumo
Certos alimentos vêm ganhando destaque na culinária mundial por apresentarem compostos bioativos com propriedades estimulantes da libido, comumente rotulados como alimentos afrodisíacos. Dessa forma, além do valor nutricional destes alimentos, surge a perspectiva associada com o bem-estar sexual de homens e mulheres. Diante disso, o objetivo desse trabalho foi analisar trabalhos pulicados sobre a influência dos alimentos afrodisíacos no desejo sexual, bem como seus efeitos em indivíduos adultos. O então estudo trata-se de uma revisão integrativa da literatura com abordagem qualitativa, que realizou análise, identificação e descrição de trabalhos, destacando a importância de pesquisas futuras sobre o tema. Foi possível observar que os alimentos tidos como afrodisíacos e seus compostos podem atuar aumentando o desejo sexual, contribuindo na melhoria da vasodilatação e na irrigação dos órgãos sexuais pela presença de nutrientes especificamente relacionados a potência sexual, sendo o consumo destes responsáveis por estimular positivamente a libido de indivíduos, como os micronutrientes zincos, magnésio, além de vitaminas do complexo B. Esses alimentos foram associados a melhora na libido, excitação, orgasmo e alguns também apresentaram ação antioxidante e anti-inflamatória. Todavia ressaltar que esses alimentos não são milagrosos, logo seu consumo deve ser feito respeitando as necessidades e individualidades nutricionais das pessoas para que assim alcancem os efeitos desejados.
\end{abstract}

Palavras-chave: Libido; Alimentos afrodisíacos; Nutrição.

\footnotetext{
Abstract

Certain foods have gained prominence in world cuisine for presenting bioactive compounds with libido-stimulating properties, commonly labeled as aphrodisiac foods. Thus, in addition to the nutritional value of these foods, there is a perspective associated with the sexual well-being of men and women. Therefore, the aim of this study was to analyze published works on the influence of aphrodisiac foods on sexual desire, as well as their effects on adult individuals.
} 
The then study is an integrative literature review with a qualitative approach, which performed analysis, identification and description of works, highlighting the importance of future research on the subject. It was possible to observe that foods considered to be aphrodisiacs and their compounds can act by increasing sexual desire, contributing to the improvement of vasodilation and irrigation of Organs sexual organs by the presence of nutrients specifically related to sexual potency, and their consumption is responsible for positively stimulating libido of individuals, such as the micronutrients zinc, magnesium, and B-complex vitamins. These foods were associated with improved libido, arousal, orgasm and some also showed antioxidant and anti-inflammatory action. However, it should be noted that these foods are not miraculous, therefore their consumption must be done respecting the nutritional needs and individualities of people so that they can achieve the desired effects.

Keywords: Libido; Aphrodisiac foods; Nutrition.

\section{Resumen}

Ciertos alimentos han ganado prominencia en la cocina mundial por presentar compuestos bioactivos con propiedades estimulantes de la libido, comúnmente etiquetados como alimentos afrodisíacos. Así, además del valor nutricional de estos alimentos, existe una perspectiva asociada al bienestar sexual de hombres y mujeres. Por tanto, el objetivo de este estudio fue analizar trabajos publicados sobre la influencia de los alimentos afrodisíacos en el deseo sexual, así como sus efectos en individuos adultos. El estudio a continuación es una revisión integradora de la literatura con enfoque cualitativo, que realizó el análisis, identificación y descripción de los trabajos, destacando la importancia de futuras investigaciones sobre el tema. Se pudo observar que los alimentos considerados afrodisíacos y sus compuestos pueden actuar aumentando el deseo sexual, contribuyendo a la mejora de la vasodilatación e irrigación de los órganos sexuales de los órganos por la presencia de nutrientes específicamente relacionados con la potencia sexual, y su consumo es responsable de estimulando positivamente la libido de los individuos, como los micronutrientes zinc, magnesio y vitaminas del complejo B. Estos alimentos se asociaron con una mejora de la libido, excitación, orgasmo y algunos también mostraron acción antioxidante y antiinflamatoria. Sin embargo, cabe destacar que estos alimentos no son milagrosos, por lo que su consumo debe realizarse respetando las necesidades nutricionales e individualidades de las personas para que puedan lograr los efectos deseados.

Palabras clave: Libido; Alimentos afrodisíacos; Nutrición.

\section{Introdução}

O termo libido provém do latim, significando desejo ou ânsia, sendo, atualmente, utilizado como um sinônimo para “desejo sexual”. Nesse contexto, a sexualidade pode ser influenciada por diversos fatores, como valores morais, culturais, religiosos ou comportamentais; o que impacta consideravelmente na libido, tanto masculina como feminina (Santos \& Gonçalves, 2014).

Ademais, o desejo sexual relaciona-se com o estado emocional do indivíduo e com a intensidade da atração física. No entanto, fatores estressantes como a depressão, ansiedade, fadiga ou o consumo de medicamentos, podem causar o declínio na libido e, consequentemente, alterar o desejo sexual dos indivíduos (Costa \& Fernandes, 2012).

Alguns alimentos vêm ganhando espaço na culinária mundial por apresentar compostos bioativos e com propriedades estimulantes da libido, comumente rotulados de “alimentos afrodisíacos". Eles se destacam, pois além do valor nutricional, existe a expectativa de potencializar o bem-estar sexual de homens e mulheres (Bona, 2013).

O termo "afrodisíaco" faz referência à deusa da mitologia grega, Afrodite, considerada deusa do amor. Na gastronomia, alguns ingredientes e receitas afrodisíacas prometem potencializar o desejo sexual em humanos. Todavia, para um prato ser considerado afrodisíaco, é preciso que ele tenha pelo menos um ingrediente estimulante da libido (Salgado, 2012; Figueredo, 2018).

A queda no desejo sexual é um problema que afeta principalmente homens obesos maiores que 40 anos. Entre outros fatores, a literatura aponta que problemas relacionados à libido podem estar relacionados à carência vitaminas e minerais. Logo, acredita-se que o consumo de alimentos-fonte desses micronutrientes podem ajudar positivamente no desejo sexual (Lima, 2015).

Em termos fisiológicos, os alimentos afrodisíacos podem aumentar a vasodilatação e favorecer a irrigação dos órgãos sexuais. Este argumento justifica-se pela presença de nutrientes especificamente relacionados à atração sexual. Outro ponto relevante é a melhora no fator psicológico, fazendo-os acreditar na ampliação das sensações (Salgado, 2012). 
Nesse contexto, torna-se importante estudar e conhecer a literatura científicas acerca da importância que o desejo sexual exerce sob à saúde física e mental de indivíduos adultos evidenciando a relação do consumo de alimentos com alegações afrodisíacas na libido. Assim, o objetivo desse trabalho foi analisar trabalhos pulicados sobre a influência dos alimentos afrodisíacos no desejo sexual, bem como seus efeitos em indivíduos adultos.

\section{Metodologia}

O então trabalho caracteriza-se como sendo um estudo secundário por meio de uma pesquisa bibliográfica, do tipo revisão integrativa. A busca de dados foi realizada nas bases de dados: Scientific Electronic Library Online (SciELO), PubMed, Literatura Latino-Americana e do Caribe em Ciências da Saúde (LILACS), utilizando os descritores nos idiomas português "Desejo Sexual", "Afrodisíacos" e "Nutrição" e inglês "Sexual desire", "Aphrodisiac" e "Nutrition".

A revisão da literatura foi realizada de forma independente, por dois autores. A coleta de dados foi norteada pela seguinte pergunta: Qual a influência dos alimentos afrodisíacos no desejo sexual?

Inicialmente, foi realizada a pré-seleção dos artigos relacionados ao tema por meio da leitura criteriosa dos títulos e resumos, e por fim foram selecionados de acordo com os critérios de inclusão e exclusão. Para a análise dos artigos, foi realizada a leitura minuciosa dos mesmos, constatando sua relação ao objetivo do presente trabalho.

Os critérios de inclusão adotados foram: artigo publicado entre os anos de 2010 e 2020 nas bases de dados científicas Scielo, Pubmed e Lilacs, ter sido divulgado em língua portuguesa ou inglesa, estar disponível na íntegra; apresentar em seus resultados fatores significativamente associados ao consumo de alimentos afrodisíacos e o desejo sexual. Seguindo critérios de exclusão: foram desconsideradas publicações referentes a teses, dissertações, resumos de congressos, anais, editoriais, comentários e opiniões, artigos de revisão.

Para detalhamento dos estudos, foram coletadas informações relevantes, entre as quais destacam-se: autores, ano de publicação, objetivo, metodologia e principais resultados. As etapas de seleção dos artigos estão descritas na figura 1.

Figura 1. Fluxograma de Seleção dos artigos.

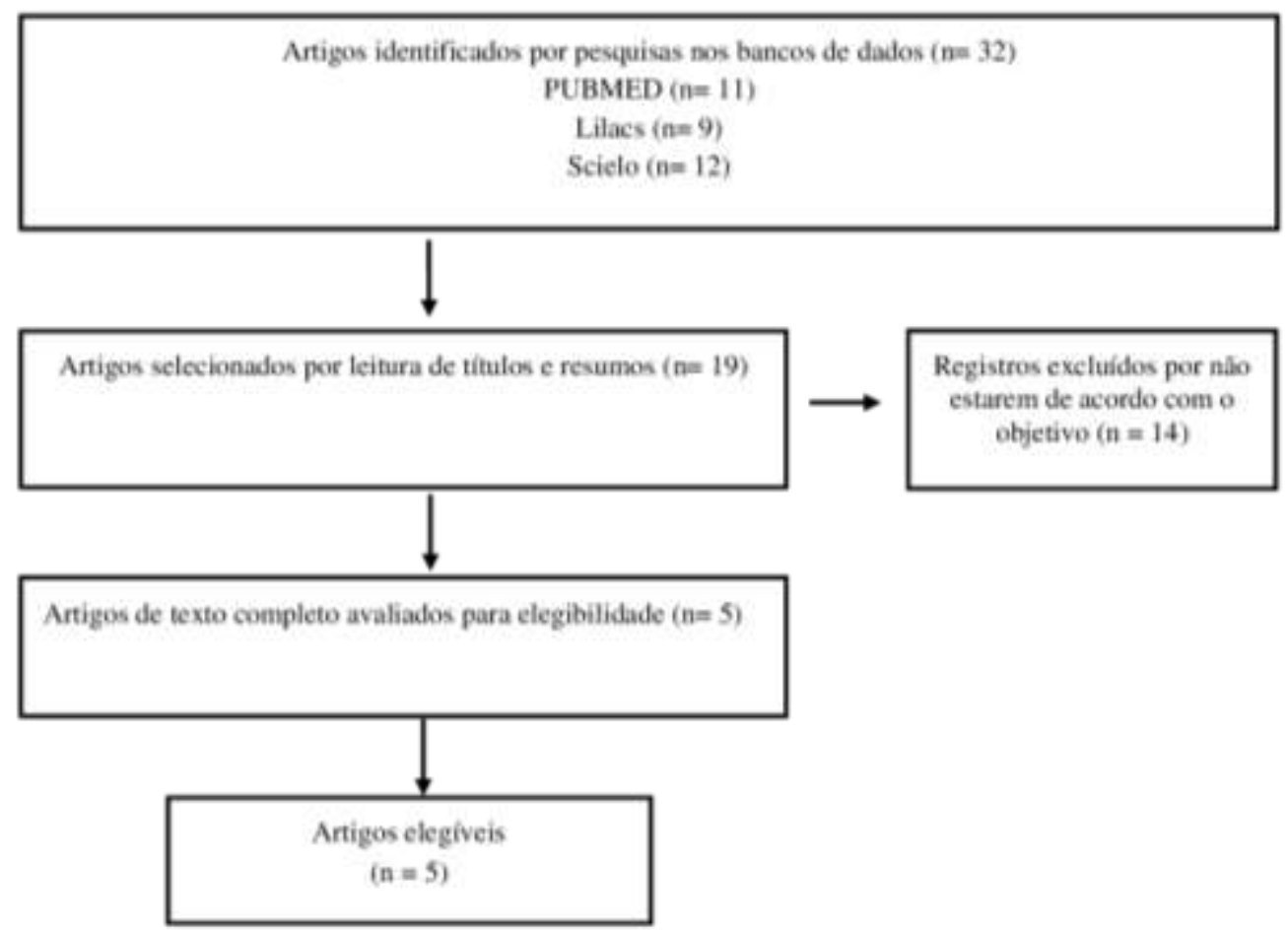

Fonte: Autores. 


\section{Resultados e Discussão}

Durante a elaboração da pesquisa obteve-se um levantamento de títulos publicados relacionados ao tema, sendo observada prevalência de publicações no idioma inglês. Com isso, obteve-se 5 publicações refinadas de acordo com os objetivos do estudo e distribuídas em diferentes periódicos. Após a análise das publicações selecionadas, fez-se o agrupamento dos estudos e síntese comparativa descrita no Quadro 1.

Quadro 1. Alimentos afrodisíacos e sua influência sob a potência sexual de indivíduos adultos.

\begin{tabular}{|c|c|c|c|}
\hline Estudos & Objetivo & Metodologia & Resultados \\
\hline $\begin{array}{ll}\text { Hessami, } & \text { et } \\
\text { al. }(2020) & \end{array}$ & $\begin{array}{l}\text { Avaliar a eficácia e } \\
\text { segurança da semente de aipo } \\
\text { para o tratamento da } \\
\text { disfunção sexual feminina. }\end{array}$ & $\begin{array}{l}\text { Este ensaio clínico paralelo, randomizado e } \\
\text { duplo-cego, } 80 \text { mulheres foram designadas } \\
\text { para receber } 500 \text { mg de semente de aipo ou } \\
\text { placebo } 3 \text { vezes ao dia por um período de } 6 \\
\text { semanas (n }=40 \text { por grupo). O questionário } \\
\text { do índice de função sexual feminina (FSFI) } \\
\text { foi usado para avaliar a função sexual das } \\
\text { mulheres antes e após o tratamento. }\end{array}$ & $\begin{array}{l}\text { Foi observado uma melhora na pontuação total do } \\
\text { FSFI que foi significativamente maior nas mulheres } \\
\text { tratadas com semente de aipo do que nas que } \\
\text { receberam o placebo. O aumento da pontuação total } \\
\text { do FSFI é contribuído principalmente pela melhora } \\
\text { nos domínios do desejo sexual, excitação, } \\
\text { lubrificação e dor no ponto final do estudo. }\end{array}$ \\
\hline $\begin{array}{l}\text { Ebrahimpour, } \\
\text { et al. (2020) }\end{array}$ & $\begin{array}{llr}\text { Avaliar clinicamente } & \text { a } \\
\text { eficácia e segurança } & \text { do } \\
\text { xarope à base de ervas } & \text { em } \\
\text { experiência } & \text { sexual } & \text { de } \\
\text { homens. } & & \end{array}$ & $\begin{array}{l}\text { O estudo foi desenhado como um ensaio } \\
\text { clínico randomizado duplo-cego. As } \\
\text { principais medidas de desfecho foram as } \\
\text { respostas obtidas com o uso da Escala de } \\
\text { Experiência Sexual do Arizona (ASEX). O } \\
\text { ASEX foi concluído por } 100 \text { homens } \\
\text { casados e aparentemente saudáveis antes e } \\
\text { depois de tomar uma dose de xarope de } \\
\text { ervas ou placebo e pelo menos uma relação } \\
\text { sexual. }\end{array}$ & $\begin{array}{l}\text { Os resultados obtidos da análise dos dados ASEX } \\
\text { mostraram que o xarope de ervas (HBS) tem um } \\
\text { efeito positivo nas experiências sexuais dos homens } \\
\text { e melhorou significativamente alguns aspectos da } \\
\text { função sexual, incluindo desejo, excitação, } \\
\text { orgasmo, ereção e satisfação. Neste estudo, a HBS } \\
\text { foi avaliada clinicamente como uma preparação } \\
\text { total e pode ser introduzida como uma alternativa a } \\
\text { outros intensificadores do desejo sexual com menos } \\
\text { efeitos colaterais }\end{array}$ \\
\hline Araújo (2013) & $\begin{array}{l}\text { Descrever os benefícios dos } \\
\text { alimentos afrodisíacos, com } \\
\text { ênfase na importância do } \\
\text { consumo e na elevação da } \\
\text { libido. }\end{array}$ & $\begin{array}{l}\text { Estudo experimental avaliativo com ênfase } \\
\text { em demostrar o potencial do consumo de } \\
\text { alimentos afrodisíacos em } 250 \text { indivíduos } \\
\text { entre os anos de } 2011 \text { e } 2012 \text {. }\end{array}$ & $\begin{array}{l}\text { Os indivíduos demostraram melhora na libido com } \\
\text { o consumo de alimentos afrodisíacos, e seus } \\
\text { componentes alimentares, fisiologicamente ativos. } \\
\text { Os compostos também têm efeito antioxidante e } \\
\text { anti-inflamatório. }\end{array}$ \\
\hline $\begin{array}{ll}\text { Dording, } & \text { et } \\
\text { al. }(2013) & \end{array}$ & $\begin{array}{l}\text { Demonstrar que a raiz de } \\
\text { maca pode ser um tratamento } \\
\text { eficaz para disfunção sexual } \\
\text { induzida por antidepressivos } \\
\text { em mulheres. }\end{array}$ & $\begin{array}{l}\text { Foi realizado um ensaio clínico duplo-cego } \\
\text { controlado por placebo de } 12 \text { semanas } \\
\text { utilizando -se de raiz de maca }(3,0 \mathrm{~g} / \mathrm{dia}) \\
\text { em } 45 \text { pacientes ambulatoriais do sexo } \\
\text { feminino com disfunção sexual cuja } \\
\text { depressão remitiu. }\end{array}$ & $\begin{array}{l}\text { Foi observado que as taxas de remissão da } \\
\text { disfunção sexual ao final do tratamento foram } \\
\text { maiores para as mulheres que receberam maca do } \\
\text { que para o grupo de placebo. Maca foi bem } \\
\text { tolerado e pode aliviar a disfunção sexual induzida } \\
\text { por antidepressivos à medida que as mulheres } \\
\text { envelhecem, particularmente no domínio do } \\
\text { orgasmo. }\end{array}$ \\
\hline $\begin{array}{l}\text { Steels, Rao \& } \\
\text { Vitetta (2011) }\end{array}$ & $\begin{array}{l}\text { Valiar o efeito do Testofen, } \\
\text { um extrato padronizado de } \\
\text { Trigonella foenum-graecum } \\
\text { (Feno-grego) e formulação } \\
\text { mineral, na libido masculina } \\
\text { em um estudo duplo cego } \\
\text { randomizado controlado por } \\
\text { placebo. }\end{array}$ & $\begin{array}{l}\text { O estudo recrutou } 60 \text { homens saudáveis com } \\
\text { idades entre } 25 \text { e } 52 \text { anos, sem disfunção } \\
\text { erétil e randomizados para uma dose oral } \\
\text { (dois comprimidos por dia) do tratamento } \\
\text { ativo ( } 600 \mathrm{mg} \text { de Testofen por dia) ou } \\
\text { placebo por } 6 \text { semanas. }\end{array}$ & $\begin{array}{l}\text { Foi observado melhora nos parâmetros específicos } \\
\text { de qualidade de vida. Testofen teve um efeito geral } \\
\text { positivo nos aspectos fisiológicos da libido. Em } \\
\text { particular, houve um aumento significativo nos } \\
\text { subdomínios de excitação sexual e orgasmo. }\end{array}$ \\
\hline
\end{tabular}

Fonte: Dados da Pesquisa.

Em seu estudo Dording, et al. (2013) sugerem que existem evidências de que pode haver fatores como os níveis de andrógenos que medeiam a eficácia do tratamento com raiz de maca no aliviar a disfunção sexual causada por antidepressivos à medida que as mulheres envelhecem, particularmente no domínio do orgasmo. Já Ebrahimpour, et al. (2020) verificaram a eficácia de xarope à base de ervas em experiências sexuais masculinas e observaram efeito positivo, com melhora significativa em alguns aspectos da função sexual, incluindo desejo, excitação, orgasmo, ereção e satisfação (Quadro 1).

Nesse contexto, os alimentos afrodisíacos são aqueles que estão relacionados à potência sexual, comprovadamente estimulantes do prazer, do estilo de vida saudável e da boa saúde mental. Nesse sentido, alguns nutrientes podem despertar a liberação de hormônios associados à felicidade como a dopamina e outros relacionados com o aumento do desejo sexual (Soares, 2012). 
Acredita-se que a queda na excitação pode estar relacionada à carência do aminoácido arginina e de alguns micronutrientes, como o zinco, selênio, vitamina $\mathrm{C}$ e folato; pois, a ingestão destes nutrientes aumenta a produção de óxido nítrico, um potente vasodilatador que estimula o desejo sexual em homens e mulheres. Os alimentos fontes destes nutrientes são o kiwi, o espinafre, a couve, a lentilha, dentre outros (Santos, 2014).

Segundo Soares (2014) para indivíduos que apresentam problemas de circulação sanguínea, determinados alimentos podem ajudar no relaxamento do tônus vascular, melhorando o fluxo de sangue para os órgãos genitais. Alimentos fonte de ácidos graxos ômega 3 como o salmão e o abacate, possuem o mesmo benefício. Outra substância que pode ajudar é a quercetina, que possui propriedades anti-inflamatórias, as quais podem melhorar o fluxo sanguíneo e, está presente em maçãs, uvas vermelhas, vinho tinto, alho e chocolate amargo.

Os alimentos afrodisíacos podem ser ingeridos individualmente ou adicionados às refeições diárias dos indivíduos, pois podem conferir sabor e valor nutritivo às refeições. São considerados alimentos afrodisíacos: o açafrão (pode deixar a região pélvica mais sensível, aumentando a sensação de prazer), o gengibre (aumenta o fluxo de sangue aos órgãos genitais e estimula o desejo sexual), o mel (estimula a síntese de hormônios sexuais que potencializam a libido), as castanhas (ajudam na lubrificação vaginal) e o alecrim (pode ser utilizado contra a impotência sexual) (Sandy, 2015).

Alimentos ricos em triptofano, um aminoácido essencial, como chocolate amargo, arroz integral, banana, quinoa, ovos e leguminosas podem auxiliar na produção de serotonina, um neurotransmissor responsável pela sensação de prazer e bemestar. Essa produção acontece a partir da hidroxilação e carboxilação do triptofano. Nesse mesmo contexto, a pimenta entra na lista dos alimentos que favorecem o apetite sexual, pois ela aumenta a frequência cardíaca, estimulando a irrigação sanguínea e quando consumida em grande quantidade, pode provocar a aumento da potência sexual (Valença, 2014).

Em seu estudo Zhang et al (2019) observaram que a carne de ostra e seus hidrolisados podem induzir atividades afrodisíacas e, consequentemente, melhorar o comportamento sexual em camundongos machos normais. Foi visto também melhora nas atividades das enzimas antioxidantes celulares endógenas e diminuindo os níveis de malonaldeído, o que pode ser útil para melhorar a função sexual. Esses resultados indicaram que as ostras podem ser um ingrediente funcional potencial com atividades antioxidantes e afrodisíacas.

\section{Considerações Finais}

Observou-se por meio dos estudos analisados, que um conjunto de alimentos estimulantes da libido, como os alimentos afrodisíacos, possivelmente estão associados a melhora no desejo sexual de indivíduos adultos. Esses alimentos foram associados a melhora na libido, excitação, orgasmo e alguns também apresentaram ação antioxidante e anti-inflamatória.

Esse efeito ocorre porque alguns nutrientes estão ligados diretamente com a potência sexual e sua ingestão ajuda a enriquecer os sentidos sexuais, porém diversos fatores devem ser considerados nessa relação com o aumento da libido: excesso de peso, estresse, depressão, ansiedade, além do consumo de alguns medicamentos, que tornam o cardápio afrodisíaco um complemento para uma vida sexualmente saudável. É importante ressaltar que, esses alimentos não são milagrosos, logo seu consumo deve ser feito respeitando as necessidades e individualidades nutricionais das pessoas para que assim alcancem os efeitos desejados.

\section{Referências}

Araújo, I. A. A., Queiroz, A. B. A., Moura, M. A. V. (2013). Representações sociais da vida sexual de mulheres no climatério atendidas em serviços públicos de saúde. Revista da Universidade Vale do Rio Verde, 22(1), 114-122.

Bona, F. D. (2013). Comer e ser comida: corpo, gastronomia e erotismo. Revista Interfaces, 19(2),189-198. 
Research, Society and Development, v. 10, n. 16, e364101622823, 2021

(CC BY 4.0) | ISSN 2525-3409 | DOI: http://dx.doi.org/10.33448/rsd-v10i16.22823

Costa, V., Fernandes, S. C. S. F. (2012). O que pensam os adolescentes sobre o amor e o sexo? Um estudo na perspectiva das representações sociais. Revista Psicologia \& Sociedade, 24(2), 391-401.

Dording, C. M., Schettler, P. J., Dalton, E. D., Parkin, S. R., Walker, R. S., Fehling, K. B., Fava, M., \& Mischoulon, D. (2015). A double-blind placebocontrolled trial of maca root as treatment for antidepressant-induced sexual dysfunction in women. Evidence-based complementary and alternative medicine: eCAM, 2015, 949036. https://doi.org/10.1155/2015/949036

Ebrahimpour, N., Khazaneha, M., Mehrbani, M., Rayegan, P., \& Raeiszadeh, M. (2020). Efficacy of Herbal Syrup on Male Sexual Experiences: A Randomized Double-Blind Clinical Trial. Journal of Traditional and Complementary Medicine, 11 (2), 103-108. https://doi.org/10.1016/j.jtcme.2020.01.004

Figueiredo, E. (2018). Prazeres da mesa, prazeres do corpo. Revista Saúde e Nutrição, 21(2), 61-68.

Hessami, K., Rahnavard, T., Hosseinkhani, A., Azima, S., Sayadi, M., Faraji, A., Emamghoreishi, M., Vafaei, H., Hessami, A., Foroughinia, L., Maasumeh, Kaviani., Roozmeh, S., \& Asadi, N. Treatment of women's sexual dysfunction using Apium graveolens L. Fruit (celery seed): A double-blind, randomized, placebo-controlled clinical trial (2021). J Ethnopharmacol, 10 (1), 264- 113400. 10.1016/j.jep.2020.113400.

Lima, A. R. L. (2015). Fatores correlacionadas a falta de potência sexual. Revista Universidade Vale do Rio Verde, 2(1), $125-136$.

Salgado, J. M. (2012). Alimentos afrodisíacos. Gazeta de Piracicaba. Nutrição e Saúde. 2(8), 56- 68.

Santos, S. M. P., \& Gonçalves, R. L. A. (2014). vivência da sexualidade por mulheres no climatério. Revista de Enfermagem, 4(1), 113-122.

Sandy, J. L. S. Libido e seus mecanismos sobre a saúde dos indivíduos. (2015). Revista da Universidade de Rio Branco. 9(2), 86-95.

Soares, D. M. R. (2012). Libido masculina e suas alterações. Revista da Universidade de Rio Verde, 5(1), 89-96.

Santos, S. M. P. S., \& Gonçalves, R. L. A. (2014). Vivência da sexualidade por mulheres no climatério. Revista Enfermagem UFSM, 4(1), 113-122.

Soares, M. C. S., \& Lima, K. M. M. (2014). Sexualidade na terceira idade: compreensão e percepção do idoso, família e sociedade. Revista da Universidade Vale do Rio Verde, 12(1), 317-326.

Steels, E., Rao, A. \& Vitetta, L. (2011). Physiological aspects of male libido enhanced by standardized Trigonella foenum-graecum extract and mineral formulation. Phytother Reserch, 25(9), 1294-1300.

Valença, C. N., Nascimento, J. M., \& Germano, R. M. (2014). Mulher no climatério: reflexões sobre desejo sexual, beleza e feminilidade. Revista Saúde e Sociedade, 19(2), 273-285.

Zhang, Z., Su, G., Zhou, F., Lin, L., Liu, X. \& Zhao, M. (2019). Alcalase-hydrolyzed oyster (Crassostrea rivularis) meat enhances antioxidant and aphrodisiac activities in normal male mice. Food Research International, 120(1), 178-187. 